\title{
Laudatio, to our beloved mentor, Professor Rudolf Slavicek
}

Rudolf Slavicek, a mentor of "modern gnathology" worldwide stays active in postgraduate education. His first scientific formation as a medical doctor (Doctor of Medicine in 1954) was followed by numerous others: his education in dentistry (certified in 1957), his post-graduation in Restorative Dentistry and Prosthetics (1958), in Orthodontics (1958-1960), but also by his passion for anatomy, anthropology, and biomechanics. This extremely broad knowledge was acquired in Vienna, Austria between 1946 (year of his baccalaureate) and 1960. On this solid foundation, he would build a professional career extraordinarily rich in innovations.

Between 1960 and 1975 he completed his knowledge by reading and meeting the great international authors in the field of oral dysfunctions. For instance, he worked with Arne G. Lauritzen, Harry C. Lundeen ... Along his private dental practice in Vienna, he developed a career as a professor-researcher during which he would demonstrate that the association between broad culture, intelligence, and willingness to share, generates extremely creative

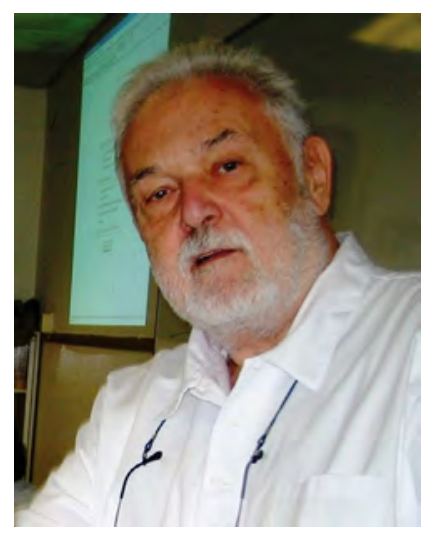
results that will significantly influence the dentistry of the late twentieth century.

\section{The scientist}

Among many publications and innovations, some major topics can be highlighted:

\section{- From anterior guidance to mandibular control}

In 1982, Rudolf slavicek presented a very extensive research work combining morphological, cephalometric, and axiographic analyses. He obtained his PhO habilitation and published his work in a first book entitled "Die funktionellen Determinanten des Kauorgans", Verlag Zahnärtzlich-Medizinisches Schrifttum München -1984. He studied the angles of the guiding inclination of the maxillary teeth. From this work on, he would largely improve the concept of canine guidance by inventing sequential guidance, functional freedom angle, retrusive control. He is the one who proposes to replace the term anterior guide by the term anterior control.

\section{- Cephalometry}

As early as the 1980s, he developed the concept of occlusal analysis superimposing cephalometric, axiographic and occlusal data following the same axis-orbital reference plane to combine sagittal condylar inclination and the inclination of the occlusal plane inclination and calculate cusp inclinations. This concept is materialized in CADIAS software.

\section{- Articulator}

At the same time, Rudolf slavicek collaborated with a new company in Munich (SAM) to develop an articulator between semi and fully adaptable. The SAM Articulator incorporates curvilinear condylar boxes and curvilinear Bennett inserts. Later he developed a new concept of condylar articulator guidance based on a hinge-axis concept which was materialized in the Reference articulator system (Gamma, Klosterneuburg, Austria).

\footnotetext{
- Axiography

It is due to the simplification of axiography that Rudolf slavicek is known internationally. This simplification was possible based on his work together with mathematician Peter Lugner on axio-pantographic principles. Originating in George G. Campion (1902), Gysi, Lee, Lundeen, SAM's axiograph system records condylar translations by eliminating condylar rotations. Rudolfslavicek was then able to develop a method of diagnostic analysis of intracapsular TMJ disorders. In the 90s, the mechanical axiographic would evolve with the
} 
development of the electronic condylography (CADIAX) developed by the Gamma company managed by his son Christian Slavicek.

\section{- Cybernetic}

As a mature scientist, having dominated the mechanical aspects, Rudolf slavicek was able to gain depth and integrate his occlusofunctional concept into the broader framework of the patient's global organism. This is materialized in the cybernetic concept of the masticatory organ published in his book "Das Kauorgan", "The Masticatory Organ" published in 2002. Consequently, Rudolf slavicek is an resolute advocate of intra- and interdisciplinary dentistry.

Recently, Professor Slavicek published a new three-part book, "Concepts in Oral Medicine" (2016) which presents many facts and documentation on the treatment of patient cases along the lines of the Vienna School of Interdisciplinary Dentistry (VieSID). In 2008, he was appointed to a new peer-reviewed journal, International Journal of Stomatology \& Occlusion Medicine (IJSOM), as a motivator and promoter for colleagues around the world, to publish their work as a forum for communication and discussion. He published hundreds of articles. He has been lecturing around the world since the 1970s. His research and articles set the standard for clinical studies in dentistry.

\section{The teacher}

His career as a member of the academia began in 1975 as an assistant at the Vienna Institute of Medicine. As of 1978, he was an Associate Professor, Head of the Prosthodontic Department at the Dental School of the University of Vienna. Since 1987, he has been Professor and Director of Fixed and Removable Prosthodontic Department, and between 1992 and 1997 he was Dean of the Dental Faculty of Vienna. He would leave the university in 1998 by inviting all his close colleagues to Vienna in a memorable farewell seminar. Yet, it was not an end, but a new beginning for a new teaching career at the Danube University in Krems. He founded the Center for Diagnosis and Therapy of Craniomandibular Functional Disorders in Vienna and he became Head of the Center for Interdisciplinary Dentistry at the Danube University, where he initiated a specialist master in oral functions-dysfunctions, as Professor Emeritus at the Danube University - Krems. His scientific research on TMJ dysfunctions (condylography), combined with cephalometrics, provides the articulator and clinical diagnosis which is unique in the world. He is the founder and mentor of the Vienna School of Interdisciplinary Dentistry in Vienna (VieSID). For many years, the training programs offered by ViesIO have been followed by a large number of colleagues from many countries around the world. He has created a real "Vienna School" in dentistry. At the same time, in 2006 he created, first in Krems and then in Vienna, the famous "Summer School" where every year, in July, his alumni meet; the fourteenth session is scheduled for July 2019. This event, currently gathering about 300 participants from 4 continents, has become a cult event of an impressive scientific level in the warm atmosphere of a real family.

\section{The man}

We know nothing about Rudolf slavicek if we miss to highlight his human qualities beside his scientific level as he skillfully combines these two facets. When you have seen Rudolf slavicek speak to his patients, teach his students, talk to his colleagues, lead an event, interact with his family members, have a smiling face for all people, you could understand the charisma that he emanates. His empathy, his attention to the others, the simplicity that he demonstrates towards everybody impress you forever. This is the indisputable mark of the great ones.

Jean-Daniel ORTHLIEB, DOS, PhO, Professor Chairperson, Vice-Dean Department of Function-Dysfunction, Imaging, Biomaterials Faculty of Odontology, Aix Marseille University, Marseille, France 